\title{
Electroconvulsive Therapy for Obesity Treatment
}

\author{
Joseph Eldor, Vladimir Kotlovker, Svetlana Kotlovker \\ Theoretical Medicine Institute, Jerusalem, Israel \\ Email: csen international@csen.com
}

Received 31 December 2013; revised 9 February 2014; accepted 25 February 2014

Copyright (C) 2014 by authors and Scientific Research Publishing Inc.

This work is licensed under the Creative Commons Attribution International License (CC BY). http://creativecommons.org/licenses/by/4.0/

(c) (i) Open Access

\begin{abstract}
ECT is most often used as a treatment for severe major depression which has not responded to other treatment, and it is also used in the treatment of mania (often in bipolar disorder), and catatonia. Its use for obesity treatment was never suggested before. However, incidental information from several studies revealed that "Five of 10 patients were obese pre-ECT, all of whom had a significant weight loss after ECT." and "Once-daily ECS over 10 days (which significantly reduced weight gain in rats)". A new hypothesis is made for the use of ECT for obesity treatment.
\end{abstract}

\section{Keywords}

Electroconvulsive Therapy (ECT); Obesity Treatment

\section{Electroconvulsive Therapy (ECT) History}

Electroconvulsive therapy (ECT), also known as electroshock, is a well-established, albeit controversial, psychiatric treatment in which seizures are electrically induced in anesthetized patients for therapeutic effect. Today, ECT is most often used as a treatment for severe major depression which has not responded to other treatment, and it is also used in the treatment of mania (often in bipolar disorder), and catatonia. It was first introduced in the 1930s and gained widespread use as a form of treatment in the 1940s and 1950s; today, an estimated 1 million people worldwide receive ECT every year, usually in a course of 6 - 12 treatments administered 2 or 3 times a week.

Ladislas J. Meduna (1896-1964) was a Hungarian neurologist and neuropathologist who discovered the first effective treatment of schizophrenia.

He first published his results in 1935 and then his major text in 1937. Die Konvulsionstherapie der Schizophrenie describes the results in 110 patients. Of these patients about half recovered. The results were much better for patients who were ill less than a year compared to those who had been ill for many years. 
A more facile form of induction of seizures, using electricity instead of chemicals, was developed by the Italian psychiatrists Ugo Cerletti and Lucio Bini. They treated their first patient with ECT in May 1938 and by the mid-1940s, electricity had replaced Metrazole as the induction agent.

\section{Electroconvulsive Therapy as Treatment for Obese Patients}

With the increased use of atypical antipsychotic medication in the treatment of mood disorders, patients are increasingly experiencing side effects, such as obesity, insulin resistance, and the metabolic syndrome, which, in turn, increases the risk of developing cardiovascular disease, type 2 diabetes, and hypertension. Maintenance electroconvulsive therapy (MECT) can be used as a prophylaxis for the recurrence of mood episodes for treatment-resistant patients. There are no reports of metabolic syndromes associated with ECT. Moss and Vaidya [1] reviewed the charts of 10 patients who have received MECT at their institution over the last 10-year period. Five of 10 patients were obese pre-ECT, all of whom had a significant weight loss after ECT. Patients whose weights were normal pre-ECT, did not experience weight loss. These finding suggests that ECT is a viable alternative for overweight patients with mood disorders who do not respond to mood stabilizers or cannot tolerate side effects.

\section{Electroconvulsive Therapy as Treatment for Obese Mice}

It has been previously suggested that low affinity [3H]mazindol binding in the hypothalamus correlates with body weight and obesity. Low affinity [3H]mazindol binding in hypothalamic crude synaptosome preparations was carried out in normoglycemic obese mice (C57 B1/6J ob/ob) as well as in their lean littermates (C57 B1/6J $+/$ ?). NIH Swiss mice were used as additional controls. Furthermore the effect on this binding site of repeated electroconvulsive shock (ECS), a treatment known to change body weight gain, was studied in rats. Neither Bmax nor Kd were altered in obese mice compared with their lean littermates or NIH Swiss mice. The obese mice had a significantly greater body weight and weight gain than either control group. Once-daily ECS over 10 days (which significantly reduced weight gain in rats) did not change binding parameters for [3H]mazindol in hypothalami. The present data do not appear to support the hypothesis that this low affinity binding site has a physiological function in the control of body weight and obesity, at least in the examined paradigm [2].

\section{Hypothalamic Obesity}

Hypothalamic obesity (HyOb) was first defined as the significant polyphagia and weight gain that occurs after extensive suprasellar operations for excision of hypothalamic tumours. However, polyphagia and weight gain complicate other disorders related to the hypothalamus, including those that cause structural damage to the hypothalamus like tumours, trauma, radiotherapy; genetic disorders such as Prader-Willi syndrome; side effects of psychotropic drugs; and mutations in several genes involved in hypothalamic satiety signalling. Moreover, "simple" obesity is associated with polymorphisms in several genes involved in hypothalamic weight-regulating pathways. Thus, understanding HyOb may enhance our understanding of "simple" obesity. HyOb is a far wider phenomenon than hitherto understood by the narrow definition of post-surgical weight gain like similarity in clinical characteristics and therapeutic approaches for $\mathrm{HyOb}$, as well as its mechanisms. HyOb, regardless of its aetiology, is a result of impairment in hypothalamic regulatory centres of body weight and energy expenditure. The pathophysiology includes loss of sensitivity to afferent peripheral humoral signals, such as, leptin on the one hand and dysfunctional afferent signals, on the other hand. The most important afferent signals deranged are energy regulation by the sympathetic nervous system and regulation of insulin secretion. Dys-regulation of 11 beta-hydroxysteroid dehydrogenase 1 (11beta-HSD1) activity and melatonin may also have a role in the development of HyOb. The complexity of the syndrome requires simultaneous targeting of several mechanisms that are deranged in the HyOb patient. Possible treatment strategies, including sympathomimetics, somatostatin analogues, triiodothyronine, sibutramine, and surgery [3].

\section{The Hypothalamus and the Regulation of Energy Homeostasis}

The hypothalamus is the focus of many peripheral signals and neural pathways that control energy homeostasis and body weight. Emphasis has moved away from anatomical concepts of "feeding" and "satiety" centres to the specific neurotransmitters that modulate feeding behaviour and energy expenditure. Williams et al. [4] have 
chosen three examples to illustrate the physiological roles of hypothalamic neurotransmitters and their potential as targets for the development of new drugs to treat obesity and other nutritional disorders. Neuropeptide Y (NPY) is expressed by neurones of the hypothalamic arcuate nucleus (ARC) that project to important appetiteregulating nuclei, including the paraventricular nucleus (PVN). NPY injected into the PVN is the most potent central appetite stimulant known, and also inhibits thermogenesis; repeated administration rapidly induces obesity. The ARC NPY neurones are stimulated by starvation, probably mediated by falls in circulating leptin and insulin (which both inhibit these neurones), and contribute to the increased hunger in this and other conditions of energy deficit. They therefore act homeostatically to correct negative energy balance. ARC NPY neurones also mediate hyperphagia and obesity in the ob/ob and $\mathrm{db} / \mathrm{db}$ mice and fa/fa rat, in which leptin inhibition is lost through mutations affecting leptin or its receptor. Antagonists of the Y5 receptor (currently thought to be the NPY "feeding" receptor) have anti-obesity effects. Melanocortin-4 receptors (MC4-R) are expressed in various hypothalamic regions, including the ventromedial nucleus and ARC. Activation of MC4-R by agonists such as alpha-melanocyte-stimulating hormone (a cleavage product of pro-opiomelanocortin which is expressed in ARC neurones) inhibits feeding and causes weight loss. Conversely, MC4-R antagonists such as "agouti" protein and agouti gene-related peptide (AGRP) stimulate feeding and cause obesity. Ectopic expression of agouti in the hypothalamus leads to obesity in the AVY mouse, while AGRP is co-expressed by NPY neurones in the ARC. Synthetic MC4-R agonists may ultimately find use as anti-obesity drugs in human subjects Orexins-A and -B, derived from prepro-orexin, are expressed in specific neurones of the lateral hypothalamic area (LHA). OrexinA injected centrally stimulates eating and prepro-orexin mRNA is up regulated by fasting and hypoglycaemia. The LHA is important in receiving sensory signals from the gut and liver, and in sensing glucose, and orexin neurones may be involved in stimulating feeding in response to falls in plasma glucose.

\section{Depression and Obesity}

Recent evidence suggests that neuronal plasticity plays an important role in the recovery from depression. Antidepressant drugs and electroconvulsive shock treatment increase the expression of several molecules, which are associated with neuronal plasticity, in particular the neurotrophin BDNF and its receptor TrkB. Furthermore, these treatments increase neurogenesis and synaptic numbers in several brain areas. Conversely, depression, at least in its severe form, is associated with reduced volumes of the hippocampus and prefrontal cortex and in at least some cases these neurodegenerative signs can be attenuated by successful treatment. Such observations suggest a central role for neuronal plasticity in depression and the antidepressant effect, and also implicate BDNF signaling as a mediator of this plasticity. The antidepressant fluoxetine can reactivate developmental-like neuronal plasticity in the adult visual cortex, which, under appropriate environmental guidance, leads to the rewiring of a developmentally dysfunctional neural network. These observations suggest that the simple form of the neurotrophic hypothesis of depression, namely, that deficient levels of neurotrophic support underlies mood disorders and increases in these neurotrophic factors to normal levels brings about mood recovery, may not sufficiently explain the complex process of recovery from depression [5].

\section{Anesthesia for Electroconvulsive Therapy in Obese Patients}

Kadar et al. [6] described obese patients who have successfully undergone over 650 consecutive uncomplicated ECT treatments without any special precautions at two major US medical centers. All of these patients were fasted overnight and breathed 100\% oxygen using a face mask before induction of anesthesia with methohexital 60 - $120 \mathrm{mg}$ IV. After loss of consciousness, succinylcholine, 40 - $120 \mathrm{mg}$ IV, was administered for muscle relaxation. Ventilation was assisted using positive pressure with a face mask until the patient was able to resume adequate spontaneous respirations. The ECT procedures were performed in the supine position with their upper body elevated 15 - 30 degrees. All patients recovered without any adverse incidents and were discharged from the recovery area within one hour after completing the ECT treatment. There were no cases of aspiration (as assessed clinically) in over 650 consecutive general anesthetics for ECT in this obese population, including 97 consecutive procedures in 9 morbidly obese (Class III) patients.

\section{Electroconvulsive Therapy in a Man with Comorbid Severe Obesity, Binge Eating Disorder, and Bipolar Disorder}

A 41-year-old man with comorbid binge-eating disorder, severe obesity, and bipolar disorder since the age of 20 
years, resistant to drug and psychotherapy combinations, worsened progressively. Relentless weight gain forced him to immobility and dependence on others. He was hospitalized for a mixed-mood episode with anxiety, mystical delusions, and auditory hallucinations. To overcome treatment resistance, he was suggested electroconvulsive therapy. After 1 electroconvulsive therapy cycle, psychological symptoms promptly improved. He received clozapine and lithium. After 2 years, he reached normal weight and fair psychopathological compensation [7].

\section{Conclusion}

A new treatment for obesity is suggested based on unnoticed information from only two articles in which "Five of 10 patients were obese pre-ECT, all of whom had a significant weight loss after ECT" and "Once-daily ECS over 10 days (which significantly reduced weight gain in rats)” as well as one case report.

Further studies are needed in order to evaluate this hypothesis.

\section{References}

[1] Moss, L.E. and Vaidya, N.A. (2006) Electroconvulsive Therapy as an Alternative Treatment for Obese Patients with Mood Disorders. Journal of ECT, 22, 223-225. http://dx.doi.org/10.1097/01.yct.0000230363.04240.9c

[2] Gleiter, C.H., Linnoila, M. and Nutt, D.J. (1989) Low Affinity Hypothalamic [3H]Mazindol Binding: A Probe for Hypothalamic Body Weight Regulation? Appetite, 12, 137-143. http://dx.doi.org/10.1016/0195-6663(89)90102-5

[3] Hochberg, I. and Hochberg, Z. (2010) Expanding the Definition of Hypothalamic Obesity. Obesity Review. http://dx.doi.org/10.1111/j.1467-789X.2010.00727.x

[4] Williams, G., Harrold, J.A. and Cutler, D.J. (2000) The Hypothalamus and the Regulation of Energy Homeostasis: Lifting the Lid on a Black Box. Proceedings of Nutrition Society, 59, 385-396. http://dx.doi.org/10.1017/S0029665100000434

[5] Castrén, E. and Rantamäki, T. (2010) The Role of BDNF and Its Receptors in Depression and Antidepressant Drug Action: Reactivation of Developmental Plasticity. Developmental Neurobiology, 70, 289-297. http://dx.doi.org/10.1002/dneu.20758

[6] Kadar, A.G., Ing, C.H., White, P.F., Wakefield, C.A., Kramer, B.A. and Clark, K. (2002) Anesthesia for Electroconvulsive Therapy in Obese Patients. Anesthesia \& Analgesia, 94, 360-361.

[7] Rapinesi, C., Del Casale, A., Serata, D., Caccia, F., Di Pietro, S., Scatena, P., Carbonetti, P., Fensore, C., Angeletti, G., Tatarelli, R., Kotzalidis, G.D. and Girardi, P. (2013) Electroconvulsive Therapy in a Man with Comorbid Severe Obesity, Binge Eating Disorder, and Bipolar Disorder. Journal of ECT, 29, 142-144. http://dx.doi.org/10.1097/YCT.0b013e31827a7f85 